\title{
Employing toxin-antitoxin genome markers for identification of Bifidobacterium and Lactobacillus strains in human metagenomes
}

\author{
Ksenia M Klimina ${ }^{\text {Corresp., }}{ }^{1,2}$, Artem S Kasianov ${ }^{1,3}{ }^{\text {, Elena U Poluektova }}{ }^{1}$ ， Kirill V Emelyanov ${ }^{3}$, Viktoriya $\mathbf{N}$ \\ Voroshilova ${ }^{3}$, Natalia V Zakharevich ${ }^{1}$, Anna V Kudryavtseva ${ }^{4}$, Vsevolod J Makeev ${ }^{1,3,4}$, Valeriy N Danilenko ${ }^{1,}$ \\ 1 Vavilov Institute of General Genetics Russian Academy of Sciences, Moscow, Russia \\ 2 Federal Research and Clinical Center of Physical-Chemical Medicine of Federal Medical Biological Agency, Moscow, Russia \\ 3 Moscow Institute of Physics and Technology, Dolgoprudny, Russia \\ 4 Engelhardt Institute of Molecular Biology, Russian Academy of Sciences, Moscow, Russia \\ Corresponding Author: Ksenia M Klimina \\ Email address: ppp843@yandex.ru
}

Recent research has indicated that in addition to the unique genotype each individual may also have a unique microbiota composition. Difference in microbiota composition may emerge from both its species and strain constituents. It is important to know the precise composition especially for the gut microbiota, since it can contribute to health assessment, personalized treatment, and disease prevention for individuals and groups (cohorts). The existing methods for species and strain composition in microbiota and not always precise and usually not so easy to use. Probiotic bacteria of the genus Bifidobacterium and Lactobacillus make an essential component of human gut microbiota. Previously we have shown that in certain Bifidobacterium and Lactobacillus species the RelBE and MazEF superfamily of toxin-antitoxin (TA) systems may be used as functional biomarkers to differentiate these groups of bacteria at the species and strain levels. We have composed a database of TA genes of these superfamily specific for all lactobacilli and bifidobacteria species with complete genome sequence and confirmed that in all Lactobacillus and Bifidobacterium species TA gene composition is species and strain specific. To analyze composition of species and strains of two bacteria genera, Bifidobacterium and Lactobacillus, in human gut microbiota we developed TAGMA software based on polymorphism in TA genes. TAGMA was tested on gut metagenomic samples. The results of our analysis have shown that TAGMA can be used to characterize species and strain of Lactobacillus and Bifidobacterium in metagenomes. 
1

2

3

4 Ksenia M. Klimina ${ }^{1,2, *}$, Artem S. Kasianov ${ }^{1,3}$, Elena U. Poluektova ${ }^{1}$, Kirill V. Emelyanov ${ }^{3}$,

5 Viktoriya N. Voroshilova ${ }^{3}$, Natalia V. Zakharevich ${ }^{1}$, Anna V. Kudryavtseva ${ }^{4}$, Vsevolod J.

6 Makeev $^{1,3,4}$, Valeriy N. Danilenko ${ }^{1,3}$

7 1Vavilov Institute of General Genetics Russian Academy of Sciences, Moscow, 119991, Russia;

$8{ }^{2}$ Federal Research and Clinical Center of Physical-Chemical Medicine of Federal Medical

9 Biological Agency, Moscow, 119435, Russia

$10{ }^{3}$ Moscow Institute of Physics and Technology, Dolgoprudny, 141701, Moscow region, Russia

$11{ }^{4}$ Engelhardt Institute of Molecular Biology, Russian Academy of Sciences, Moscow, 119991, 12 Russia; 


\section{Abstract}

15 Recent research has indicated that in addition to the unique genotype each individual may also

16 have a unique microbiota composition. Difference in microbiota composition may emerge from

17 both its species and strain constituents. It is important to know the precise composition especially

18 for the gut microbiota, since it can contribute to health assessment, personalized treatment, and

19 disease prevention for individuals and groups (cohorts). The existing methods for species and

20 strain composition in microbiota and not always precise and usually not so easy to use. Probiotic

21 bacteria of the genus Bifidobacterium and Lactobacillus make an essential component of human

22 gut microbiota. Previously we have shown that in certain Bifidobacterium and Lactobacillus

23 species the RelBE and MazEF superfamily of toxin-antitoxin (TA) systems may be used as

24 functional biomarkers to differentiate these groups of bacteria at the species and strain levels. We

25 have composed a database of TA genes of these superfamily specific for all lactobacilli and

26 bifidobacteria species with complete genome sequence and confirmed that in all Lactobacillus and

27 Bifidobacterium species TA gene composition is species and strain specific. To analyze composition of species and strains of two bacteria genera, Bifidobacterium and Lactobacillus, in human gut microbiota we developed TAGMA software based on polymorphism in TA genes. TAGMA was tested on gut metagenomic samples. The results of our analysis have shown that

31 TAGMA can be used to characterize species and strain of Lactobacillus and Bifidobacterium in metagenomes. 


\section{$34 \quad$ Background}

The human gastrointestinal tract is a habitat for a wide variety of microorganisms, mainly bacteria $[1,2]$. Human gut microbiota consists of $10^{14}$ cells of more than 1000 species. $[3,4]$. The gut microbiota has been described as a new endocrine organ that plays an important role in formation and maintenance of immunity and overall homeostasis, including formation of neuropsychological and behavioral features [5]. An individual metagenome depends on the country, urban or rural dwelling, age group, diet preferences, and general health state or various diseases [6]. The gut microbiota of healthy adults (from 2.5 to 65 years) is quite stable [7]. Individual differences of gut microbiota $(\mathrm{GM})$ and their effect on the macroorganism depend on the microbiome composition not only at the level of phyla or species but also at the strain level. Sub-species variates of bacterial strains can display a substantial variation in metabolism type or other properties $[8 ; 9]$. Recently it has been reported that the presence of particular strains of the same species correlates with the onset and progression of human disorders including adiposity and insulin resistance [10]. It is important to know the precise composition especially for the gut microbiota, since it can contribute to health assessment, personalized treatment, and disease prevention for individuals and groups (cohorts). However, human microbiota composition is usually analyzed at the family or genus level rather than at the species and strains level. Methods for strain characterization in microbiota are still not adequate. Most of software tools based on the presence of marker genes like MetaPhlAn [11] or MG-RAST [12] have resolution at most at the species level. Approaches based on gene copy number variation [9] require very deep metagenome sequencing $(>500 \mathrm{x})$. We believe that identification of novel functional markers highly represented in most human gut microbiome samples and using them to characterize the bacteria of the GM 
56 microbiota at the strain level would make an important contribution into analysis of metagenome 57 samples.

Toxin-antitoxin gene systems (TASs) are present in the genomes of the overwhelming majority of bacteria and archaea $[13,14]$. They are involved in bacterial persistence, antibiotic tolerance, stress response, apoptosis, biofilm formation $[15,16]$. Type II TASs are most numerous and well-studied. They usually consist of two components, the toxin (T) and antitoxin (A). Usually toxin and antitoxin genes are located nearby and form an operon. The toxin causes death of cells or suppresses their proliferation; the antitoxin interacts with the toxin blocking its activity. Toxins target at mRNAs, ribosomes, DNA-gyrases, cytoskeletal proteins and cell wall synthesis systems. The antitoxin is less stable than the toxin. Under suppression of transcription or translation the antitoxin is digested by proteases to release free toxin leading to cell death or growth arrest [17]. Previously we showed that genes of the MazEF and RelBE superfamilies of type II TAS were present in all tested strains of several species of Bifidobacterium and Lactobacillus and demonstrated how TA genes could be used to identify species and strains in these bacteria $[18,19$, 20].

Bifidobacterium and especially Lactobacillus make a small part of human gut microbiome. However, they are cultivated and well studied bacteria that have health-promoting properties and contribute to homeostasis of the host $[21,22]$. It has been reported that individual gut microbiomes include strains of Bifidobacterium and Lactobacillus specific for the individual, which agrees with the general pattern of individual strain preference observed for many bacterial taxa [23, 24]. Bacterial strains administered to animals (rodents) and humans substantially influenced the microbiota composition, as well as metabolomic and immunity processes, ultimately affecting the 
78 disease development. The effect substantially depended on the particular bacterial strain being

79 administered, including Bifidobacterium and Lactobacillus strains [23, 24].

80 We suppose that TASs can provide additional functional markers for metagenomic analysis

81 of species and strain diversity of the genera Lactobacillus and Bifidobacterium [25]. To this end,

82 we created a database of MazEF and RelBE chromosomal toxin and antitoxin genes in all complete

83 genomes of Bifidobacterium and Lactobacillus genus and TAGMA software which conducts

84 species and strain identification. We tested TAGMA to identify species and strains of lactobacilli

85 and bifidobacteria in 147 metagenome samples from the Human Microbiome Project (available in

86 the Human Microbiome Project database (https://www.hmpdacc.org/HMASM/, subtab "stool"))

87 as well as in 5 in-house samples (see Methods: In-house metagenome characterization). The results

88 were compared with those obtained with other programs, PhymmBL and MetaPhlAn. Based on a

89 limited number of well selected markers TAGMA displays performance at least comparable to

90 that displayed by those programs, may be somewhat underperforming PhymmBL. TAGMA can

91 identify species of lactobacilli and bifidobacteria displaying prediction quality comparable to that

92 of PhymmBL and MetaPhlAn but it is based on the small number of carefully selected markers,

93 which thus can be obtained with very deep targeted sequencing, and also it works much faster than

94 PhymmBL or MetaPhLan. In some cases TAGMA also can identify individual bacterial strains,

95 the option, which is not implemented in MetaPhlAn or PhymmBL. 
97

98

99

100

101

102

103

104

105

106

107

108

109

110

111

112

113

114

115

116

117

118

119

\section{Methods}

\section{Software for metagenomic analysis}

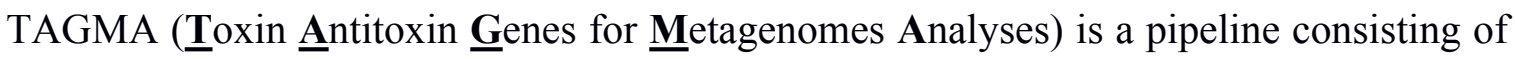
existing published software and in-house scripts [https://github.com/LabGenMO/TAGMA]. In the first step the algorithm scans BLASTN alignments of TAS and identifies markers (substitutions and indels) that distinguished gene variants, identified by all to all BLASTN alignments. In the second step metagenomic reads are aligned with TAS genes using BowTie2 [26] algorithm. In the third step the aligned reads that support particular marker variants are identified.

Since some marker position can be not covered by reads, while some other could appear due to sequencing errors, the discernibility matrix $\mathrm{G}(\mathrm{g} \times \mathrm{g})$ is constructed in the fourth stage. Here $\mathrm{g}$ is the set of detected genes or gene variants, $\mathrm{G}(1,2)=1$ means that gene 1 cannot be distinguished from gene 2 (0 otherwise). Due to fragmental read coverage some genes that are theoretically distinguishable by the complete marker set become indistinguishable with the observed marker set. The TAGMA reports such cases and outputs the smallest possible group of genomes, that can still be distinguished with the observed set of markers (Fig. 1).

In the fifth step $\operatorname{St}(s \times s)$, the strain discernibility matrix is build. Here $s$ is the number of strains that have at least one detected genetic marker. For instance, $\operatorname{St}(3,4)=1$ means that strain number three is not distinguishable from strain number four. This matrix is not symmetric. One cannot distinguish strain 1 from strain 2 if the coverage of all detected genes in strain 1 is not enough to distinguish variants of these genes from those in strain 2 (or if these genes are completely identical). But there are cases when strain 2 can be distinguished from strain 1 if strain 2 contains another set of detected genes or at least one gene variant that has a characteristic marker position. In this case one is not allowed to consider strain 1 as a false positive, because the genomes in 
120 metagenomic samples are not fully covered with reads and information can be missing due to 121 sequencing depth deficiency.

122 In the sixth step, sets of indiscernible strains are derived from the strain discernibility 123 matrix. The number of strains that cannot be distinguished from each other and from the target 124 strain is used as the measure of performance. This measure is lower for better detected strains.

125 TAGMA can be used for identification of Lactobacillus and Bifidobacterium species and 126 individual strains in metagenomes.

Collection and annotation of TAS type II genes in Lactobacillus and Bifidobacterium

128

129

130

131

132

133

134

135

136

137

138

139

140

141

\section{known genes}

First, we have collected a compilation of known genes of listed TASs type II superfamilies MazEF and RelBE in Lactobacillus rhamnosus, L. casei, L. helveticus, L. plantarum, Bifidobacterium longum (from NCBI Gene database [http://www.ncbi.nlm.nih.gov/gene]) and used it for gene annotation. Then for extended annotation of toxin and antitoxin genes we used the Lactobacillus and Bifidobacterium strains with complete genome sequence. We identified homologous regions in all complete genomes of Bifidobacterium (53 complete genomes) and Lactobacillus (72 complete genomes) (data available in NCBI [http://www.ncbi.nlm.nih.gov/genome]), located open reading frames and checked for proteins corresponding to annotated genes. Homologous sequences were identified with TBLASTX aligner with e-value threshold $10^{-20}$, protein coding reading frames were predicted with GeneMarkS algorithm [http://exon.gatech.edu/GeneMark/] [27], whereas protein sequences were assessed with InterPro [http://www.ebi.ac.uk/interpro/] [28]. For target regions we adopted those in which homologous sequence overlapped with gene predicted by GeneMakrS for more than $80 \%$. We 
142 considered only TASs located at chromosomes only, not at plasmids. All gene locus tags (748) are

143 available at https://github.com/LabGenMO/TAGMA.

$144 \quad$ Heatmap construction

145 We used BLASTN to classify all genes into groups. Genes from the same group were

146 considered as identical if they were similar for more than $88 \%$. Each group of genes had a set of

147 SNPs distinguishing it from representatives of other groups. 49353 nucleotide sequences of 148 plasmids were downloaded from NCBI by using entrez query "NUCCORE:plasmid[Title]".

149 Nucleotide sequences of TASs genes were mapped by BLASTN program at the plasmid 150 sequences. The hits were filtered with identity threshold values at $80 \%$. If a plasmid hit was located

151 for a TASs gene hit, we assumed that this TA system gene could be transferred by plasmids. A set

152 of Python scripts tagma.py (https://github.com/LabGenMO/TAGMA) was developed to assess the

153 presence of genes of different groups in the selected strains and species. These groups of genes

154 were used to construct heatmaps with the help of seaborn python library

155 [https://stanford.edu/ mwaskom/software/seaborn/].

Phylogenetic analysis of metagenomic samples

157

To analyze diversity of species in metagenomic samples we used two software packages

158

159

160

161

162

163

164 on alignment of metagenomic reads with clade-specific marker genes. We used it with the default set of parameters. In contrast to MetaPhlan, PhymmBL uses BLAST [30] aligner to map reads or assembled sequences on sequences of whole bacterial genomes. To prepare the data for PhymmBL, we used metaVelvet, the de novo metagenome assembler [31], with the following parameters: the kmer value 55, the minimum contig length $1000 \mathrm{bp}$. PhymmBL software was used with default parameters. 


\section{In-house metagenome characterization}

We used the gut metagenomes (feaces), isolated from people living in the Central region

167

168

169

170

171

172

173

174

175

176

177

178

179

180

181

182

183

184

185

186

187

of Russia. All five metagenomes have been deposited in GenBank (NCBI) under the accession no.: SRX1869839 (RM1, a healthy 6 year old girl); SRX1869842 (RM2, a healthy 28 year old woman), SRX1870055 (RM3, a healthy 34 year old woman), SRX1878777 (RM4, a healthy 28 year old woman), SRX1878778 (RM5, a 51 year old men with type II diabetes mellitus (DM), the sugar level of 8.7).

\section{DNA extraction and quantification}

DNA was extracted from the feaces of metagenomes (RM1-RM5) using the QIAamp DNA

Stool Mini Kit (Qiagen, Germany) according to the manufacturer protocol. The gDNA quantity was determined on the Qubit 2.0 Fluorometer (Invitrogen, USA) per manufacturer instructions.

\section{Fragmentation of DNA}

Each of the gDNA samples were fragmented by nebulization using compressed nitrogen gas, nebulizers, nebulization buffer (Illumina, USA) and glycerol (Sigma, USA). Fragmented DNA samples were purificated using the MinElute PCR Purification Kit (Qiagen, Germany) according to the manufacturer protocol. Nebulization was performed for the duration of one minute with nitrogen (pressure 2.1 bar) with a total input of $1 \mathrm{mg}$ gDNA for each sample. The final size of fragmented gDNA samples was determined on Agilent 2100 Bioanalyzer (Agilent, USA) per manufacturer guide and was approximately of 300-500 bp.

\section{Illumina library preparation and sequencing}

Fragmented and cleaned DNA samples were prepared using TruSeq DNA LT Sample Prep Kit (Illumina, USA) following the TruSeq DNA Sample Preparation v2 Guide starting with the “Perform End Repair" step. In brief, fragmented DNA samples were end repaired, 3' ends were 
188 adenylated and TruSeq adapters were ligated to the each gDNA sample. The in-Line Control DNA 189 was added to each enzymatic reaction. Libraries were then size-selected using a $2 \%$ low range 190 ultra-agarose gel with $1 \mathrm{X}$ TAE buffer run at $120 \mathrm{~V}$ for $120 \mathrm{~min}$. The $500-600 \mathrm{bp}$ fragments (of 191 which $\sim 120$ bp were the ligated adapters) were cut out with a sterile scalpel blade for each 192 individual sample. Each gDNA sample was purified using the MinElute Gel Extraction Kit 193 (Qiagen, Germany) following manufacturer instructions. Then after gel extraction, the libraries 194 were subjected to amplification by means of PCR process according to the manufacturer protocol 195 (10 cycles). The libraries were validated by visualization on the Agilent 2100 Bioanalyzer 196 (Agilent, USA), quantified using qPCR. The libraries were then sequenced as 2x250 bp paired197 end runs on the Illumina MiSeq Systems (Illumina, USA) according to the manufacturer 198 instruction. Raw sequencing reads were obtained using Illumina analysis software (MiSeq 199 Reporter, Illumina, USA). Trimming v0.3 program [32] was used for trimming, FastQC v.0.10.1 200 [33] program was used for quality control. Chimeric reads where filtered with Uchime [34] 201 algorithm (which is a part of Userach v7.0 program). Classification of bacteria was made with 202 RDP MultiClassifier v1.1 program [35]. 
204 Results

205

206

207

208

209

210

211

212

213

214

215

216

217

218

219

220

221

222

223

224

225

226

\section{Distribution of TASs genes in Bifidobacterium and Lactobacillus sp.}

Previously, we showed that different L. rhamnosus and L.fermentum strains have different sets of TASs from RelBE and MazEF superfamilies. The same was shown for genus Bifidobacterium. Primary investigation showed that genes of these families are found in all studied Lactobacillus and Bifidobacterium species [18, 19, 36]. Now we investigated how the TASs of RelBE and MazEF superfamilies are distributed in other species of Lactobacillus and Bifidobacterium stored in the GenBank. For this purpose, genes of MazEF and RelBE superfamilies were annotated (see Methods) and a database of toxins and antitoxins genes has been constructed. The same TA genes may be present on the chromosome as well as, rather rare, on a plasmid [37]. Our objective was to create a database containing only the chromosomal TA genes. For this purpose, we identified plasmid regions similar to TA genes located in genomes. From all TA genes 14 were found to have plasmid homologs and have been removed from our database. Figure 2 displays the dot matrix showing the presence or absence of the TA genes in the species of Lactobacillus and Bifidobacterium.

All studied species, except L. paracasei, have species-specific genes. These genes in $L$. paracasei are the same as in L. casei. These species are very close and difficult to differentiate from each other [38]. Distribution of almost any gene reveals some degree of species specificity (Fig. 2). In some cases, closely related species of Lactobacillus (L. johnsonii - L. gasseri; L. helveticus - L. acidophilus - L. amylovorus) share common genes (Fig. 2A). Several common genes have also been found in some bifidobacterial species: B. longum, B. bifidum, B. breve, $B$. adolescentis and B. kashiwanohense (Fig. 2B). B. longum and B. breve belong to the "B. longum group", B. adolescentis and B. kashiwanohense belong to the "B. adolescentis group" and $B$. 
227 bifidum belong to the "B. bifidum group". These species groups are always closely located in the 228 phylogenetic tree and are more remote from other groups of bifidobacteria, indicating their 229 common separation from other groups, and their subsequent divergence. Nevertheless, each 230 species has its own specific set of TA genes.

We performed similar analysis for strains. Distribution of toxins and antitoxin genes is

232

markedly strain specific (see Fig. S1). Strains belonging to the same species of bacteria have similar but not identical sets of $\mathrm{T}$ and A genes. Consequently, these genes can be used to identify species and strains of Lactobacillus and Bifidobacterium. This property can be used to characterize the presence of specific strains of Lactobacillus and Bifidobacterium species in human microbiota.

\section{The software}

The general idea of our approach is the same as in other techniques of metagenomic profiling (16sRNA, MetaPhlAn, PhymmBL). Each strain is associated with the specific set of genetic markers. We use two types of markers: i) TA genes themselves (each strain has different set of TA genes, so particular TA genes found in the sample help to identify the strain); ii) differences in the sequences of particular TA genes (each strain has pattern of point variants and indels in its TA genes).

One of the main difficulties of metagenomic analysis is the fragmented structure of genomic data. Only parts of some genes are present in the sample reads; reads for other genes can be totally missing. These difficulties may be counteracted by deeper sequencing of a small set of selected markers. An example of such marker is ribosomal RNA genes, which is efficient for species identification, although it is not always possible to distinguish between similar species but is less efficient for identification of strains. Thus, a broader set of markers is necessary for strain identification, which can include makers either present or missing in different strains, which thus 
250 can be identified by marker combinations. Additionally, the target sequences can be variable

251 enough, to use specific single nucleotide for strain characterization. Both properties are valid for

252 genes belonging TAS families: these systems may be present of absent in different strain genomes

253 and their genes are not very conservative.

254 Our approach to metagenomic analysis consists in two stages. In the first stage all known

255 genomes of the studied subgroup are compared to evaluate possible variable TAS-related genetic

256 markers, and assess occurrence of different variants in the strains. At this stage all sequences are

257 aligned against each other (all-over-all blast) and the differences between genome sequences are

258 identified. Genetic markers thus become grouped into homogeneous groups and intragene variants

259 (point substitutions and indels) that distinguish genomic sequences in each group. The

260 metagenome is analyzed in the second stage. All reads are mapped against genetic markers with

261 Bowtie2 program. Algorithm tracks mismatches that occur while alignment metagenomic reads

262 and genetic markers, if mismatch occurs in the position that distinguishes two homogeneous

263 genetic markers, such marker is discarded. To allow for the fragmented structure of metagenome

264 coverage with the reads we considered possible marker dropouts due to low read coverage. The

265 entire pipeline is called TAGMA ( $\underline{\text { Toxin }}$ Antitoxin Genes for Metagenomes Analyses) and is 266 available at [https://github.com/LabGenMO/TAGMA].

$267 \quad$ Metagenomic analysis

268

To test our software, we studied representation of Lactobacillus and Bifidobacterium

species in metagenomes. First, we analyzed five metagenomes, RM1-RM5 studied in our Lab and obtained from people of different age and state of health (see the Methods). The faecal samples

271 has been collected in the identical conditions, DNA was extracted and libraries have been prepared. 
273 and Bifidobacterium genera (Table 1). To validate that Lactobacillus and Bifidobacterium species

274 were present in the microbiomes we plated feces suspension on nutrient media (data unpublished).

275 Lactobacilli was found in RM1 in much lesser quantities than in other metagenomes, which is the

276 known feature of children microflora [39].

277 We applied TAGMA for analysis of five in-house human gut metagenomes up to the level

278 of species; the results were compared with those obtained using MetaPhlan2 and PhymmBL (Table

279 2). PhymmBL uses BLAST to align sequences of complete metagenomes against genomes of 280 prokaryotes. MetaPhlAn performs read clustering before doing BLAST searches, thus increasing 281 time efficiency. The analysis showed that those species of Bifidobacterium, which were detected

282

283

284

285

with both PhymmBL and MetaPhlan2, were discovered by TAGMA. Lactobacillus genus was present in very low quantities (Table 1) and it was difficult to detect the corresponding species. Those species of Bifidobacterium and Lactobacillus which were not detected by TAGMA were either species that were present in metagenome in small amounts (less than $0.4 \%$ ) or that had no markers with sufficient read coverage.

Table 2 illustrates that the TAGMA gives more information than MetaPhlan2 but less than PhymmBL. But the advantage of TAGMA is that it can analyze metagenome up to strain level (Table S1, S2) if the metagenome contains a specific set of TA genes or at least to the level of a group of strains if these strains contain identical T and A genes (see Table S1, S2).

Then we tested TAGMA on the open access data. For the analysis, we selected 147 samples of the intestinal metagenomes of healthy human subjects, available in the Human Microbiome Project database (https://www.hmpdacc.org/HMASM/, subtab "stool"). These samples were isolated from the feces of healthy men and women aged 18 to 40 years old living in the United States. Previously, Kovtun et al. analyzed these 147 samples. In their analysis, they searched the 
296 metagenomes for bacterial enzymes involved in the synthesis of neuroactive compounds and 297 determined the taxonomic composition using both Kraken and Metaphlan2 programs. Their 298 analysis confirmed the presence of lactobacilli and bifidobacteria in the metagenomes (at the 299 species level) [40]. Using TAGMA, we identified Lactobacillus and Bifidobacterium in each 300 metagenome down to the strain level (Table S3). Table S3 shows the strains (or groups of strains) 301 of Lactobacillus and Bifidobacterium that satisfied the following conditions: the TA genus 302 coverage is more than $60 \%$ and the number of markers detected in a strain more than two (or $50 \%$ ).

303 The composition and abundance of Lactobacillus and Bifidobacterium strains were significantly 304 different between all the metagenomes. 


\section{Discussion}

307 Two main approaches are currently used to characterize taxonomic diversity in 308 metagenome samples: the whole metagenome shotgun analysis and analysis of PCR amplicons 309 from the ribosomal 16S RNA gene [41]. Lactobacilli and bifidobacteria constitute only a small 310 part of human gut microbiota $(0,5$ and $10 \%)$ [42], therefore the existing methods not always can 311 adequately assess the presence of these genera (especially lactobacilli). Identification of species 312 and strains in microbiota is even more difficult. Yet, the results of metagenome analysis at this 313 level make a useful characteristic of individual microbiota.

In this report, we show that genes of type II TASs can be used as functional markers for computer assisted species and strains characterization of lactobacilli and bifidobacterial in human gut microbiota. The database of toxins and antitoxins genes for these two genera of bacteria has been created and it has been shown that distribution of TAS is species- and strain specific. developed and tested on 5 metagenomic samples. It turned out that TAGMA could effectively identify Lactobacillus and Bifidobacterium species and sometimes their individual strains in the metagenome; in some cases, TAGMA identified specific groups of strains. TAGMA can be used

322 for characterization of individual metagenomes or groups of metagenomes (for example, people from different habitats). TAGMA was compared with existing methods of metagenomic analysis

324 (MetaPhlan, PhymmBL), and at the species level outperformed MetaPhlan2 but proved to be less sensitive than PhymmBL. This difference can be explained by the fact that each program uses

326 different markers to identify the genus and species of bacteria. The most comprehensive 327 information of species composition in metagenomes is revealed using several programs. 


\section{Conclusion}

329 In contrast to other programs, based on a large set of genomic markers (up to 1 million 330 [https://bitbucket.org/biobakery/metaphlan2], TAGMA employs only a small set of genes (from 2 331 to 10) to determine species in metagenomes. Therefore, TAGMA is much more time efficient, it 332 takes only several hours to analyze one metagenome, whereas MetaPhlan2 and PhymmBL analysis 333 takes several days on one computational core of desktop computer. The developed software can 334 be also applied for metagenomic analysis of oral and vaginal cavity, where lactobacilli are 335 dominating. We plan to expand the TASs database not only for analysis of the strain diversity of 336 the Lactobacillus and Bifidobacterium species but also for other bacteria found in the 337 gastrointestinal tract of the

human. 


\section{Declarations}

339 Acknowledgments

340 This work was performed using the sequencing equipment of EIMB RAS “Genome" center

341 (http://www.eimb.ru/rus/ckp/ccu_genome_c.php) and VIGG RAS «Genetic Polymorphisms»

342 center (http://vigg.ru/institute/ckp/ckp-obn-ran-geneticheskii-polimorfizm/) for the opportunity to

343 use computational resources. 


\section{REFERENCES}

1. Montiel-Castro, A.J., González-Cervantes, R.M., Bravo-Ruiseco, G., Pacheco-López, G. The microbiota-gut-brain axis: neurobehavioral correlates, health and sociality. Front Integr Neurosci. 2013;7:16.

2. Thakur, A.K., Shakya, A., Husain, G.M., Emerald, M., Kumar, V. Gut-Microbiota and Mental Health: Current and Future Perspectives. J Pharmacol Clin Toxicol.2014;2:1016.

3. Browne, H. P., Forster, S. C., Anonye, B. O., Kumar, N., Neville, B. A., Stares, M. D., Goulding D., and Lawley T.D. Culturing of 'unculturable' human microbiota reveals novel taxa and extensive sporulation. Nature. 2016; 533:543-546.

4. Rajilić-Stojanović, M., de Vos, W.M. The first 1000 cultured species of the human gastrointestinal microbiota. FEMS Microbiol Rev. 2014;38:996-1047.

5. Flint, H.J., Scott, K.P., Duncan, S.H., Louis, P., Forano, E. Microbial degradation of complex carbohydrates in the gut. Gut Microbes. 2012;3:289-306.

6. Lloyd-Price, J., Abu-Ali, G., Huttenhower, C. The healthy human microbiome. Genome Med. 2016;8:51.

7. Faith, J.J., Guruge, J.L., Charbonneau, M., Subramanian, S., Seedorf, H., Goodman, A.L., Clemente J.C., Knight R., Heath A.C., Leibel R.L., Rosenbaum M., and Gordon J.I.. The long-term stability of the human gut microbiota. Science. 2013;341:1237439.

8. Zhu, A., Sunagawa, S., Mende, D.R., Bork, P. Inter-individual differences in the gene content of human gut bacteria species. Genome Biol. 2015; 16:82.

9. Greenblum, S., Carr, R., Borenstein, E. Extensive Strain-Level Copy-Number Variation across Human Gut Microbiome Species. Cell. 2015;160:583-594. 
367

368

369

370

371

372

373

374

375

376

377

378

379

380

381

382

383
10. Zhang, C., Zhao, L. Strain-level dissection of the contribution of the gut microbiome to human metabolic disease. Genome Med. 2016;8:41.

11. Segata, N., Waldron, L., Ballarini, A., Narasimhan, V., Jousson, O., Huttenhower, C. Metagenomic microbial community profiling using unique clade-specific marker genes. Nature Methods. 2012;9:811 - 814.

12. Keegan K.P., Glass E.M., Meyer F.. MG-RAST, a Metagenomics Service for Analysis of Microbial Community Structure and Function. Methods Mol Biol. 2016;1399:207-33.

13. Unterholzner, S.J., Poppenberger, B., Rozhon, W. Toxin-antitoxin systems: Biology, identification, and application. Mob Genet Elements. 2013:3:e26219.

14. Klimina K.M., Poluektova E.U., Danilenko V.N. Bacterial toxin-antitoxin systems: properties, functional significance, and possibility of use (review). Applied Biochemistry and Microbiology. 2017;53;494-505.

15. Van Melderen, L. Toxin-Antitoxin Systems: Why so Many, What for? Cur Opin Microbiol. 2010;13:781-785.

16. Hu, Y., Benedik, M.J., Wood, T.K. Antitoxin DinJ influences the general stress response through transcript stabilizer CspE. Environ Microbiol. 2012;14:669-679.

17. Yamaguchi, Y., Park, J.H. and Inouye, M. Toxin-Antitoxin Systems in Bacteria and Archaea. Ann Rev Genet. 2011;45:61-79.

18. Klimina, K.M., Kjasova, D.Ch., Poluektova, E.U., Krügel, H., Saluz, H.P., Danilenko, V.N. Identification and characterization of Toxin-Antitoxin systems in strains of Lactobacillus rhamnosus, isolated from humans. Anaerobe. 2013;22:82-89. 
388

389

390

391

392

393

394

395

396

397

398

399

400

401

402

403

404

405

406

407

408
19. Averina, O.V., Alekseeva, M.G., Abilev, S.K., Il'in, V.K., Danilenko, V.N. Distribution of genes of toxin-antitoxin systems of mazEF and relBE families in bifidobacteria from human intestinal microbiota. Genetika. 2013;49:315-327.

20. Krügel H., Klimina K.M., Mrotzek G., Tretyakov A., Schöfl G., Saluz H.P., Brantl S., Poluektova E.U., Danilenko V.N. Expression of the toxin-antitoxin genes yefMLrh, yoeBLrh in human Lactobacillus rhamnosus isolates. Journal of basic microbiology. (2015) 55 (8), 982-991.

21. Turroni, F., van Sinderen, D. and Ventura, M. Genomics and Ecological Overview of the Genus Bifidobacterium. In J Food Microbiol. 2011;149:37-44.

22. Walter, J. Ecological role of lactobacilli in the gastrointestinal tract: implications for fundamental and biomedical research. Appl Environ Microbiol. 2008;74:4985-4996.

23. Derrien M., van Hylckama Vlieg J.E. Fate, activity, and impact of ingested bacteria within the human gut microbiota. Trends Microbiol. 2015;23(6):354-66.

24. Saez-Lara M.J., Gomez-Llorente C., Plaza-Diaz J., Gil A. The role of probiotic lactic acid bacteria and bifidobacteria in the prevention and treatment of inflammatory bowel disease and other related diseases: a systematic review of randomized human clinical trials. Biomed Res Int. 2015;2015:505878.

25. Klimina K., Poluektova E., Kudryavtseva A., Kasianov A., Danilenko V. Type II toxinantitoxin systems for metagenomic studies. FEBS OPEN BIO (2018) 8, 451-451

26. Langmead, B. \& Salzberg, S. L. Fast gapped-read alignment with Bowtie 2. Nat Methods. 2012;9:357-359. 
409

410

411

412

413

414

415

416

417

418

419

420

421

422

423

424

425

426

427

428

429
27. Besemer, J., Lomsadze, A., Borodovsky, M. GeneMarkS: a self-training method for prediction of gene starts in microbial genomes. Implications for finding sequence motifs in regulatory regions. Nucleic Acids Res. 2001;29:2607-2618.

28. Jones, P., Binns, D., Chang, H.Y., Fraser, M., Li, W., McAnulla, C., McWilliam H., Maslen J., Mitchell A., Nuka G., Pesseat S., Quinn A.F., Sangrador-Vegas A., Scheremetjew M., Yong S.Y., Lopez R., Hunter S. InterProScan 5: genomescale protein function classification. Bioinformatics. 2014;30:1236-1240.

29. Brady, A. and Salzberg, S.L. PhymmBL expanded: confidence scores, custom databases, parallelization and more. Nature Methods. 2011;8;367.

30. Altschul, S.F., Gish, W., Miller, W., Myers, E.W., Lipman, D.J. Basic local alignment search tool. J Mol Biol. 1990;215:403-410.

31. Namiki, T., Hachiya, T., Tanaka, H., Sakakibara, Y. MetaVelvet: An extension of Velvet assembler to de novo metagenome assembly from short sequence reads. Nucleic Acids Res. 2012;40:e155.

32. Bolger, A. M., Lohse, M., \& Usadel, B. Trimmomatic: A flexible trimmer for Illumina Sequence Data. Bioinformatics. 2014;30:2114-20

33. Ramirez-Gonzalez, R.H., Leggett, R.M., Waite, D., Thanki, A., Drou, N., Caccamo, M., Davey R. StatsDB: platform-agnostic storage and understanding of next generation sequencing run metrics. F1000Res. 2013;2:248.

34. Edgar, R.C., Haas, B.J., Clemente, J.C., Quince, C., Knight, R. UCHIME improves sensitivity and speed of chimera detection. Bioinformatics. 2011;27:2194-2200. 
430

431

432

433

434

435

436

437

438

439

440

441

442

443

444

445

446

447

448

449

450

451

452

35. Wang, Q., Garrity, G.M., Tiedje, J.M., Cole, J.R. Naive Bayesian classifier for rapid assignment of rRNA sequences into the new bacterial taxonomy. Appl Environ Microbiol. 2007;73:5261-5267.

36. Poluektova EU, Yunes RA, Epiphanova MV, Orlova VS, Danilenko VN. The Lactobacillus rhamnosus and Lactobacillus fermentum strains from human biotopes characterized with MLST and toxin-antitoxin gene polymorphism. Arch Microbiol. 2017; doi:10.1007/s00203-017-1346-5.

37. Remisetti B.C., Santhos R.S. Horizontal gene transfer of chromosomal Type II toxinantitoxin systems of Escherichia coli. FEMS Microbiol Lett. 2016;363(3).

38. Wuyts S., Wittouck S., De Boeck I., Allonsius C.N., Pasolli E., Segata N., Lebeer S. Largescale phylogenomics of the Lactobacillus casei group highlights taxonomic inconsistencies and reveals novel cladeassociated features. mSystems. 2017;2:e00061-17.

39. Botina S.G., Klimina K.M., Koroban N.V., Amerkhanova A.M., Zinchenko V.V., Danilenko V.N. Revised Classification of Native Probiotic Strains of Lactobacillus Used in Russian Federation. Russian journal of genetics. 2010. 46.11 1485-1492

40. Kovtun A.S., Averina O.V., Zakharevich N.V., Kasianov A.S., Danilenko V.N. In Silico Identification of Metagenomic Signature Describing Neurometabolic Potential of Normal Human Gut Microbiota. Genetika, 2018, 54, 9, 1-11

41. Jovel, J., Patterson, J., Wang, W., Hotte, N., O'Keefe, S., Mitchel, T., Perry T., Kao D., Mason A.L., Madsen K.L., Wong G.K. Characterization of the Gut Microbiome Using 16S or Shotgun Metagenomics. Front Microbiol. 2016;7:459.

42. Zhernakova, A., Kurilshikov, A., Bonder, M.J., Tigchelaar, E.F., Schirmer, M., Vatanen, T., Mujagic Z., Vila A.V., Falony G., Vieira-Silva S., Wang J., Imhann F., Brandsma 
453 E., Jankipersadsing S.A., Joossens M., Cenit M.C., Deelen P., Swertz M.A.; LifeLines 454 cohort study, Weersma R.K., Feskens E.J., Netea M.G., Gevers D., Jonkers D., Franke 455 L., Aulchenko Y.S., Huttenhower C., Raes J., Hofker M.H., Xavier R.J., Wijmenga C., Fu 456 J. Population-based metagenomics analysis reveals markers for gut microbiome 457 composition and diversity. Science. 2016;352:565-569. 


\section{$459 \quad$ Figure legends}

460 Figure 1. The algorithm of the TAGMA software.

461 Figure 2. Representation of TASs type II genes of superfamilies RelBE and MazEF in the species 462 of Lactobacillus (A) and Bifidobacterium (B). Black boxes show the presence of a gene. The name 463 of each group of TA genes consists of the name of a gene and the number of a group.

Additional files

466 Table S1 Strain diversity of Bifidobacterium in metagenomes.

467 Table S2 Strain diversity of Lactobacillus in metagenomes.

468 Table S3 Strain diversity of Lactobacillus and Bifidobacterium in the samples of the intestinal 469 metagenomes (from the Human Microbiome Project database).

470 Figure S1 Representation of TASs type II genes of superfamilies RelBE and MazEF in

471 Lactobacillus strains (A) and Bifidobacterium strains (B). Black boxes show the presence of a 472 gene. The name of each group of TA genes consists of the name of a gene and the number of a 473 group. 


\section{Table $\mathbf{1}$ (on next page)}

The presence of genera Bifidobacterium and Lactobacillus in the metagenomes determined by MetaPhlan2 and PhymmBL programs

The presence of genera Bifidobacterium and Lactobacillus in the metagenomes determined by MetaPhlan2 and PhymmBL programs. Ph - PhymmBL, Mt - MetaPhIAn2 


\begin{tabular}{|l|c|c|c|c|c|c|c|c|c|c|}
\hline & \multicolumn{2}{|c|}{ RM1 } & \multicolumn{2}{c|}{ RM2 } & \multicolumn{2}{c|}{ RM3 } & \multicolumn{2}{c|}{ RM4 } & \multicolumn{2}{c|}{ RM5 } \\
\cline { 2 - 12 } & Ph & Mt & Ph & Mt & Ph & Mt & Ph & Mt & Ph & Mt \\
\hline Lactobacillus sp & 0.1 & - & 1.32 & - & 0.17 & - & 0.92 & - & 2.33 & 2,66 \\
\hline Bifidobacterium sp & 1.61 & 5.17 & 2.79 & 12.77 & 8.31 & 23.63 & 1.77 & 10.47 & 2.43 & 5.74 \\
\hline
\end{tabular}




\section{Table 2 (on next page)}

The presence Bifidobacterium sp. and Lactobacillus $s p$. in 5 in-house metagenomes detected by three different programs

The presence Bifidobacterium sp. and Lactobacillus sp. in 5 in-house metagenomes detected by three different programs 


\begin{tabular}{|c|c|c|c|}
\hline Species & PhymmBL & MetaPhlAn2 & TAGMA \\
\hline \multicolumn{4}{|c|}{ RM1 } \\
\hline B.adolescentis & + & + & + \\
\hline B.bifidum & + & + & + \\
\hline B.longum & + & + & + \\
\hline B.breve & - & - & + \\
\hline L.rhamnosus & - & - & + \\
\hline \multicolumn{4}{|c|}{ RM2 } \\
\hline B.adolescentis & + & + & + \\
\hline B.angulatum & - & + & + \\
\hline B.bifidum & + & + & + \\
\hline B.longum & + & + & + \\
\hline B.breve & + & - & + \\
\hline B.dentium & + & - & - \\
\hline L. delbrueckii & + & - & - \\
\hline L. fermentum & + & - & - \\
\hline L. salivarius & + & - & - \\
\hline \multicolumn{4}{|c|}{ RM3 } \\
\hline B.adolescentis & + & + & + \\
\hline B.bifidum & + & - & - \\
\hline B.breve & + & - & + \\
\hline B.catenulatum & - & + & - \\
\hline B.dentium & + & - & - \\
\hline B.longum & + & + & + \\
\hline B.pseudocatenulatum & - & + & + \\
\hline B. kashiwanohense & - & - & + \\
\hline \multicolumn{4}{|c|}{ RM4 } \\
\hline B.adolescentis & + & + & + \\
\hline B.bifidum & + & + & + \\
\hline B.longum & + & + & + \\
\hline L.fermentum & + & - & - \\
\hline L.rhamnosus & - & - & + \\
\hline \multicolumn{4}{|c|}{ RM5 } \\
\hline B.adolescentis & + & + & + \\
\hline B.animalis & + & - & - \\
\hline B.bifidum & + & - & - \\
\hline L.ruminis & + & + & + \\
\hline L.fermentum & + & - & \\
\hline
\end{tabular}


Figure 1

The algorithm of the TAGMA software

The algorithm of the TAGMA software 


$$
G=\left(\begin{array}{lllll}
1 & 1 & 0 & 1 & 0 \\
0 & 1 & 0 & 0 & 0 \\
1 & 0 & 1 & 0 & 1 \\
1 & 0 & 1 & 1 & 0 \\
1 & 0 & 1 & 0 & 1
\end{array}\right) \quad S t=\left(\begin{array}{llll}
1 & 1 & 0 & 1 \\
0 & 1 & 1 & 0 \\
1 & 0 & 1 & 1 \\
0 & 1 & 0 & 1
\end{array}\right)
$$

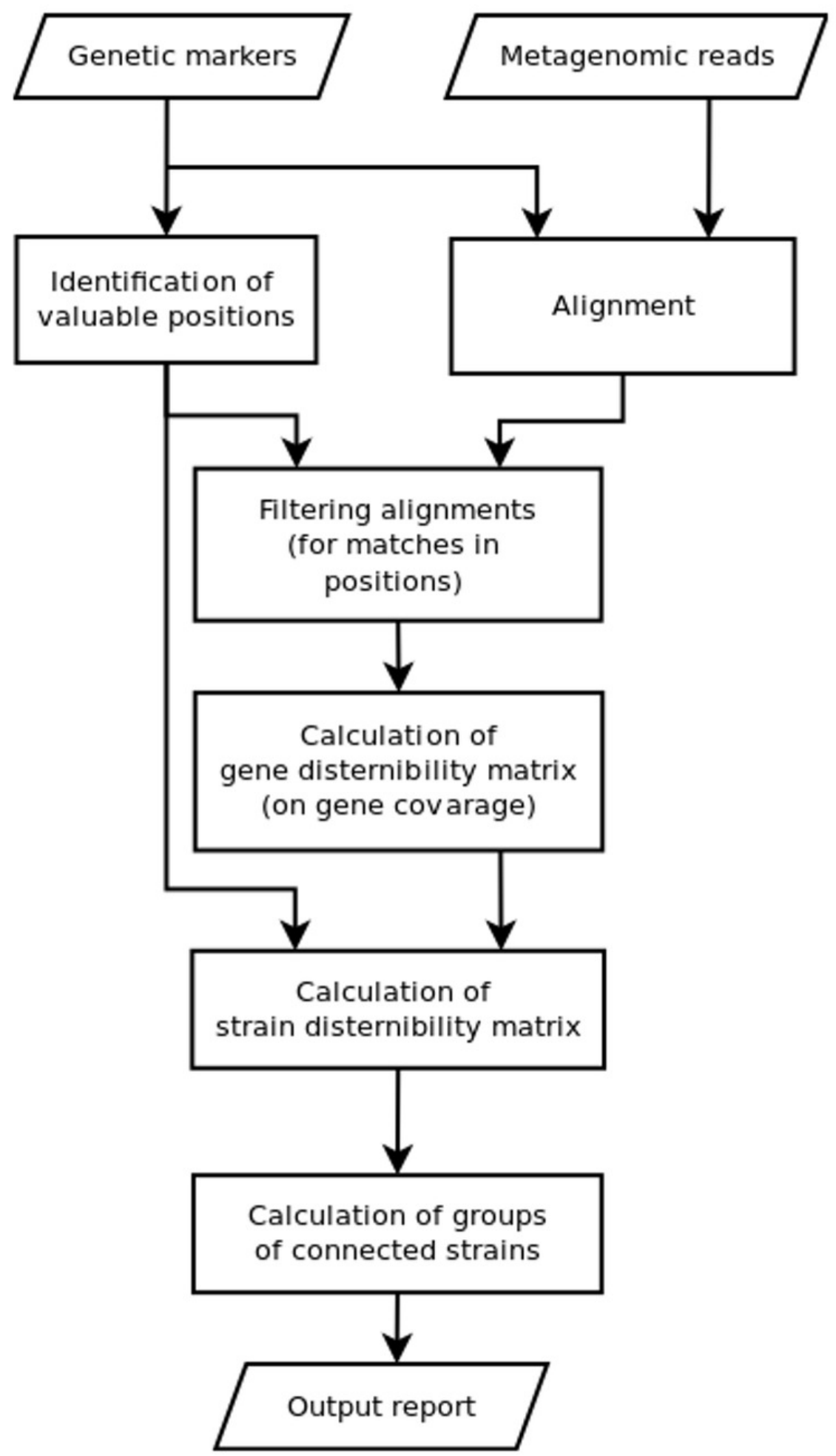


Figure 2

Representation of TASs type II genes of superfamilies RelBE and MazEF in the species of Lactobacillus (A) and Bifidobacterium (B)

Representation of TASs type II genes of superfamilies RelBE and MazEF in the species of Lactobacillus (A) and Bifidobacterium (B). Black boxes show the presence of a gene. The name of each group of TA genes consists of the name of a gene and the number of a group

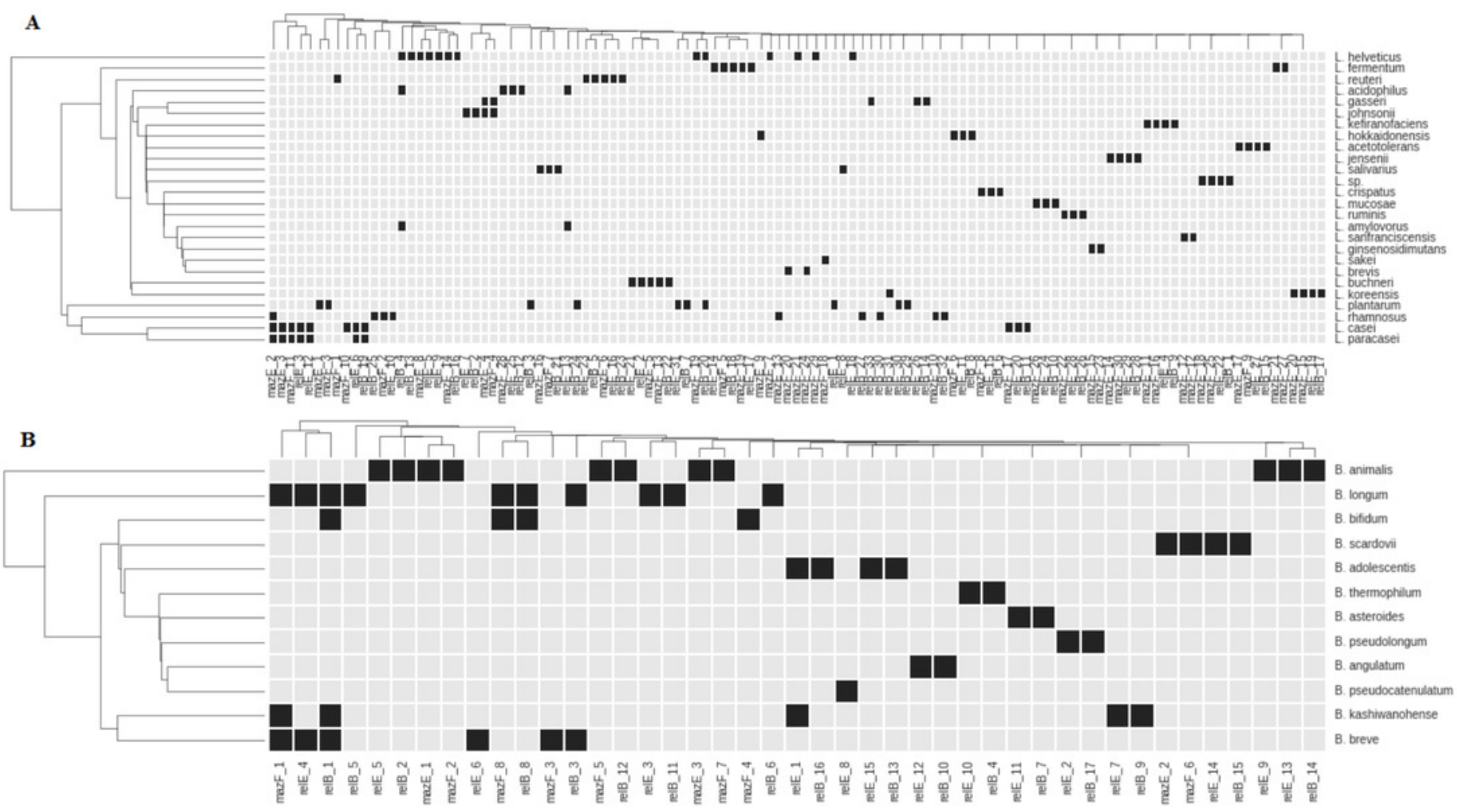

